\title{
The Role of Warranty of Misinforming for New Product Adoption
}

\author{
Dimitar Christozov \\ American University in Bulgaria, Blagoevgrad, Bulgaria \\ dgc@aubg.bg \\ Stefanka Chukova \\ Victoria University of Wellington, New Zealand \\ stefanka.chukova@vuw.ac.nz

\begin{abstract}
Plamen Mateev
Sofia University "St. Kliment Ohridski”, Sofia, Bulgaria

pmat@fmi.uni-sofia.bg
\end{abstract}

\begin{abstract}
This study considers the impact of warranty agreement during the process of a new product adoption, mainly its impact on the risk of misinforming, which naturally arises during this process. It addresses the risk of misinforming in the following two phases of the product adoption - acquiring and utilization of the product. In the first phase the warranty of misinforming acts as a promotional tool, while in the second phase it serves mostly as a risk sharing mechanism. The paper investigates the role of warranty of misinforming in an "ideal" case, when the consumer optimizes the purchase decision led by rational assessment of his needs, product's price and some supplementary costs.
\end{abstract}

Keywords: warranty, information asymmetry, risk, misinforming, purchase decision, acquiring, utilization, optimization.

\section{Introduction}

The current development of worldwide markets can be characterized with an increasing complexity and multi-functionality of their goods. Smart phones replaced the old-fashion mobile devices, which very recently replaced the traditional land phones. Selection, purchasing and utilization of a new device are no longer simple, routine tasks. The increased product complexity requires relevant customer expertise to make proper use of modern devices. The adoption process of new

Material published as part of this publication, either on-line or in print, is copyrighted by the Informing Science Institute. Permission to make digital or paper copy of part or all of these works for personal or classroom use is granted without fee provided that the copies are not made or distributed for profit or commercial advantage AND that copies 1) bear this notice in full and 2) give the full citation on the first page. It is permissible to abstract these works so long as credit is given. To copy in all other cases or to republish or to post on a server or to redistribute to lists requires specific permission and payment of a fee. Contact Publisher@InformingScience.org to request redistribution permission. products has attracted the attention of several researchers and it has been studied from different perspectives. Eng and Quaia (2009) review the findings about new performance in the literature and conceptualize a framework and its related propositions for improving new product adoption process. Manchanda, Xie, and Youn (2009) identify that the two main influences leading to adoption at the individual consumer 
level are marketing communication and interpersonal communication. Bhagat, Goyal, and Lakshmanan (2012) present a problem formulation to study the impact of user's experience (or projected experience) with a product in the adoption process. Sethi, Prasad, and He (2008) propose a model for new-product adoption that incorporates price and advertising effects. An optimal control problem that uses the model as its dynamics is solved explicitly to obtain the optimal price and advertising effort over time. Parry, Cao, and Song (2011) deal with forecasting the adoption of innovative products. They examine the usefulness of a probabilistic neural network algorithm for forecasting new product adoption and compare their approach with the standard forecasting methods. Devos, Van Landeghem, and Deschoolmeester (2012) consider the problem of smalland medium-sized enterprises with their lack of IT proficiency and dependence on external IT expertise, and the role of information asymmetry in the acquisition of strategic IT.

Also, the increased complexity and multi-functionality of the products lead to an increased level of information asymmetry during the purchasing process, which may mislead customers in their purchase decision making. Often, the customer buys a product, which he/she doesn't need or pays for product features that are not needed and will never be used. On the other hand, new devices provide not only challenges, but also opportunities for extending the set of problems the customer may wish to solve with. Nowadays, every purchase of a new product requires making decision under uncertainty.

This risk - the risk of misinforming, caused by the information asymmetry, is based on the inability of the customers to acquire complete, accurate, and sufficient understanding regarding the features and constraints of a new product. This paper discusses the role the warranty, especially the warranty of misinforming, may play not only in making purchase decision, but also in the process of product utilization.

We investigate the role of warranty of misinforming in an "ideal" case, when the consumer optimizes the purchase decision led by rational assessment of his/her needs, product's price and some supplementary costs. Also, in this "ideal" case, we consider the product's quality to be positively correlated with the product's price. Under these assumptions, the role of warranty during product adoption process is discussed by addressing the following questions:

- What are the misinforming risks and how the warranty of misinforming may affect the adoption process?

- How such risks can be quantified, so to allow proper justification of the product warranty?

- What is the adoption cost and how to optimize the adoption process based on this cost?

The paper is organized in four sections. We start with describing the framework of this study. The second section discusses the role of warranty during the acquiring phase and the third section addresses the utilization phase. The fourth section defines measures for quantifying the cost of adoption, and presents a formulation of an optimization problem reflecting the tradeoff between quality and price in a new product purchase decision making process.

\section{Basic Concepts of New Product Adoption}

\section{New Product Adoption Process}

The process of adoption of a new product includes two phases - acquiring and utilization, which can be further divided into a set of activities. The phase of acquiring of a new product includes the following stages:

a) identification of the customer's needs;

b) setting criteria for product acceptance, including the quality thresholds; 
c) collecting information regarding the alternative options;

d) selecting the most appropriate option;

e) product purchasing.

The phase of the product utilization includes the following stages:

i. learning how to accomplish the intended set of tasks with the new product;

ii. learning what additional tasks can be accomplished with the new product;

iii. learning which tasks cannot be accomplished with the new product.

During the first phase information asymmetry may cause misunderstanding of the actual properties of the product, which may lead to wrong purchase decisions in two aspects:

- to buy a product, which doesn't allow to accomplish the intended tasks and does not meet customer's needs;

- not to buy a suitable for the customer's tasks and needs product.

The role of warranty in acquiring a new product was introduced in Christozov, Chukova, and Mateev $(2006,2007)$. The purchase decision is a tradeoff between product's quality and product's price. The higher quality means higher probability that the product will address the customer's needs, but also implicates higher product price.

During the utilization phase of the new product - the customer learns how to use the new product. The risk in this phase is two-fold. Firstly, the customer may recognize that the product is not suitable for accomplishing all of his/her tasks, but also that there are other products in the market that are more suitable, than the chosen one.

\section{Informing Aspects of New Product Adoption: Misinforming}

In general, the phases of the product adoption, as outlined above, are itself informing processes. The first phase can be considered as process of learning via communication (talking to the seller, reading messages regarding the product) and the second phase - as learning via accumulating own experience while using the product. From this point of view, the second phase can be considered as a verification of what was learned during the first phase regarding customer's needs and how the selected product is suitable for them.

\section{Warranty of Malfunctioning and Warranty of Misinforming}

The two types of risks related to purchasing and utilizing a new product are related to the following probabilities: (1) the probability of buying a faulty product; (2) the probability of buying a product that is not suitable for the buyer's needs, i.e., the customer has been misinformed regarding the product features. These two risks are subject of two types of warranty agreements (see Christozov, Chukova, and Mateev, 2009) - warranty of malfunctioning and warranty of misinforming. Malfunctioning refers to inability of the product to perform its functions as intended and as it is stated in the product description. The risks associated with malfunctioning are well defined and studied within reliability theory, quality control, etc. The models for assessing warranty cost and establishing an "optimal" in some sense warranty policy have been studied over the last 30-40 years and they are well understood and analyzed (see Blischke and Murthy, 1996).

Misinforming refers to inability of the customer to obtain a complete understanding on the product's features and properties at the time of the purchase. Initially, the aim of offering warranty to cover this risk was mainly to assure product promotion and not to address this risk itself. The risk of misinforming exists in any trading, in particular, it increases significantly in an indirect commerce, e.g., in e-business. This risk is even higher when dealing with high complexity 
products, such as computers or "smart" devices. The successful estimation of the probability of misunderstanding requires the development of new methods and approaches on measuring and quantifying the risk of misinforming. Nowadays only a few researchers have addressed this phenomenon and more research is needed to develop appropriate tools for dealing with these issues.

\section{The Role of Warranty of Misinforming in the Product Acquiring Process}

The success in the phase of acquiring of a new product depends on the successful communication between sellers, offering their products, and buyers examining how suitable the product is for their needs. During this communication two-sided information asymmetry can be detected - the seller is an expert regarding the product specifications and the buyer is fully aware of his/her needs and how the product will be used to address these needs at the time of the purchase. The seller provides a message, describing the product, without a complete knowledge of the recipient's needs and his/her ability to understand correctly the enclosed information; the buyer receives the message and interprets it according to his/her level of expertise. This often leads to misunderstanding, which we call "misinforming" and consider it as a failure in the informing communication process.

Besides the information asymmetry, the buyer's decision is always influenced by the uncertainty regarding the product specifications as well as his/her "wish list" set of tasks to be performed using the product. The buyer doesn't know exactly what additional opportunities the new product may offer, e.g., what additional tasks could be supported by the product and what product's "quality" will be needed for successful accomplishment of these new, unknown at the time of the purchase, tasks.

In the phase of new product acquiring, the initial four stages a) - d), listed in the previous section are affected by the aforementioned information asymmetry as follows:

a) In the first stage, in order to identify his/her own needs, the customer have to be aware what is offered on the market and what tasks these products can support. Identifying the needs of a buyer is equivalent to matching the buyer's tasks to products' specifications as described in the seller's ads.

b) During the second stage a set of criteria with corresponding quality thresholds are identified, so that the product which satisfies them is acceptable for the buyer. The successful completion of the second stage requires that the customer is able to understand most of the technical details regarding how his/her tasks can be accomplished with the product. Identifying the acceptable price is among the important criteria for products' suitability.

c) The stage of collecting information regarding alternative options requires the matching of the products' advertised specifications with the criteria of product suitability and selecting the options that satisfy the pre-specified "quality" thresholds. This is the stage of creating the "short list" of products for possible purchase.

d) Choosing the product, which provides the best match for the set of buyer's product suitability criteria, is equivalent to solving an optimization problem, which reflects the buyer's uncertainty in purchase decision making.

Often the uncertainty in purchase decision making is not clearly understood by the customers, because of disregarding the impact of information asymmetry in obtaining and interpreting information. If the impact of information asymmetry is disregarded, the risk of being misinformed is underestimated and often ignored in purchase decision making. In data collected in an empirical study, published in Christozov, Chukova, and Mateev (2007) among 65 respondents only 6 
recognized their uncertainty of whether the product is suitable for their needs. The data revealed that the overall risk of making a wrong decision was above $40 \%$.

Warranty of misinforming provides the customer with a risk free trial period when an unsuitable product could be returned with no penalties. During this trial period the uncertainties regarding the suitability of the product can be resolved to some extent. The existence of warranty of misinforming allows the customers to choose the cheapest product from the "short list" of suitable products, and if it meets the customer's needs, then this purchase decision is the best (optimal) purchase decision from customer viewpoint.

\section{The Role of Warranty of Misinforming in the Process of Utilization of a New Product}

The process of learning about the actual properties and features of the newly acquired product and how this product can be efficiently utilized is usually quite lengthy. It includes consulting and reading about product specifications, but mostly it is a sequence of trials and errors while experimenting with the product. The goal of this process is to reduce uncertainty regarding usability of the acquired product. During this phase there are two groups of activities and consequently two sources of risks:

- Learning how to accomplish initially identified tasks in efficient manner with the new product. The discovery that the product is not suitable for some of the intended tasks or at least learning that this cannot be done efficiently is one of the major outcome, indicating the failure in the process of acquisition. On one hand, this activity leads to reducing the uncertainty regarding the properties of the product, and on the other hand - it reduces the uncertainty regarding the actual qualities needed for accomplishing efficiently the intended tasks.

- Learning about the additional opportunities the new product is able to support. The realization that there are additional options that the new product may provide could lead to dissatisfaction with the purchase decision made. For example, the next generation gadget may allow the accomplishment of a numerous additional tasks, not identified at the time of purchase. This reduces the uncertainty regarding the customer's own needs and extends the understanding of the product quality, and related price criterion.

The warranty of misinforming promotes the emphasis on the evolving, potential needs when making purchase decision. It offers opportunities to adjust customer needs to the best practice and to select the product with best suitability for accomplishing the initially identified tasks as well as for tasks evolved during the trial period.

\section{Costs of Product Adoption}

\section{The Framework}

Any rational, informed, optimal purchase decision making for a new product should aim to minimize costs and maximize profit. The cost components that we will take into account are as follows:

- Direct costs - the price of the product.

- Indirect costs - time and efforts for learning the product features and how to accomplish tasks in an efficient manner, utilization. This cost is recovered by the future usage of the product. 
- Sunk costs - time and efforts for identifying the needs and the set of the product acceptance criteria; mapping products' properties to the acceptance criteria when making the purchase decision. This cost cannot be recovered.

Also, the benefits of the purchase decision that we will take into account are as follows:

- Monetary benefits or profit - savings for accomplishing tasks with the product, which otherwise will be carried out by an external service for higher cost.

- Non-monetary benefits - the ability to accomplish tasks independently, without relying on external services.

The aim of a product purchase decision is to accomplish a certain set of tasks. The learning aspect during the adoption process identifies a set of additional new tasks that can be performed with the product, as well as a set of tasks, that cannot be reasonably accomplish with the new product. The following simple example illustrates this idea. Let us consider purchasing a home printer. The printer's productivity, measured by number of printed pages per minute, is the "quality" measure of this product. At the time of the purchase, the customer plans to print all of his/her printing jobs. The purchased printer allows printing all kind of printing jobs, which do not exceed 10-15 pages per job. Any job with more than twenty pages may take a long time, so that its completion at home is inconvenient. For large jobs, the customer could use external services incurring associated charges.

\section{Quality of Acceptance and Buyer's Cost}

In general, the optimization problem of making a purchase decision can be defined as follows:

A customer (buyer) $b$ needs to perform a set of tasks $\left\{a_{i}^{k}\right\}$, where $\boldsymbol{i}$ corresponds to the categories of tasks $\left\{A_{i}\right\}$ the product $\boldsymbol{D}$ is suitable for, and $\boldsymbol{k}$ is the index for the different tasks within the category $A_{i}$ the buyer $b$ needs to address. For each of these $a_{i}^{k}$ tasks $b$ needs a product with given quality level $q_{i}^{k}$. The parameter $q_{i}^{k}$ can be normalized, so that its values are within the interval $[0,1]$. The properties/features of the product $\boldsymbol{D}$ can be measured by the probability that it allows the buyer to accomplish tasks at a given quality level.

A product $\boldsymbol{D}$ is characterised by a parameter $p_{i}$ that represents the quality level of $\boldsymbol{D}$ with respect to category $A_{i}$. Then for a given set of tasks $\left\{a_{i}^{k}\right\}$ from $A_{i}, \boldsymbol{D}$ could be suitable for some of these and not suitable for others. For the case, illustrated in Fig. 1, the product $\boldsymbol{D}$ is suitable for accomplishing tasks with the following quality levels $-q_{i}^{1}, q_{i}^{5}$ and $q_{i}^{3}$, and not suitable for tasks with quality levels $q_{i}^{2}$ and $q_{i}^{4}$ for $b$ 's tasks from category $A_{i}$.

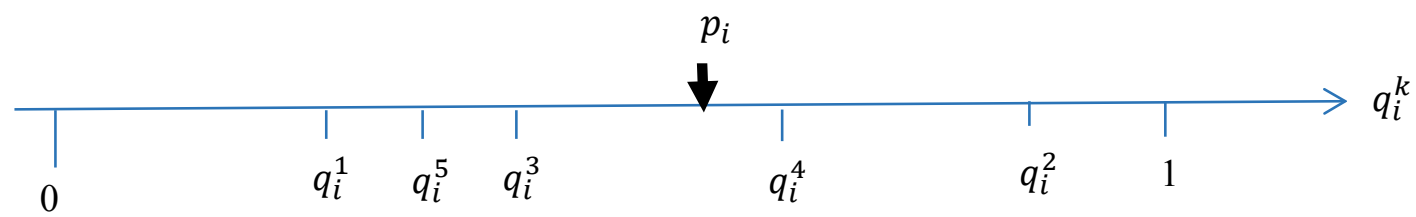

Fig. 1. An illustration of the suitability of product $\boldsymbol{D}$ to accomplish tasks $a_{i}^{k}$

We call the parameter $p_{i}$ the "quality level of product $\boldsymbol{D}$ for category $A_{i}$ " and $q_{i}=\max \left\{q_{i}^{k}: q_{i}^{k}<p_{i}\right\}$ the "quality level for category $A_{i}$ for customer $b$ ". Also, we assume that $c_{i}$ is the cost (price) to get this quality level $q_{i}$. For Fig. $1, c_{i}$ is the price of a product capable of accomplishing all tasks, which require quality $p_{i}$ and therefore can accomplish all tasks, which need quality less than $p_{i}$, i.e., tasks $-a_{i}^{1}, a_{i}^{5}$ and $a_{i}^{3}$. On the other hand, this product is not suitable for accomplishing tasks, which require quality $q_{i}^{4}, q_{i}^{2}$ or higher. We denote the price of prod- 
uct $\boldsymbol{D}$ by $c_{D}=c_{p_{i}}$, i.e., the price of the product is equivalent (or measured by) to the price of quality level of tasks the product is suitable for. Next, we denote by $C_{i}^{k}$ the quality cost (price) needed to accomplish a task the product $\boldsymbol{D}$ is not suitable for. The $\operatorname{cost} C_{i}^{k}$ corresponds to the price of quality required for a task $a_{i}^{k}$, which accomplishment requires quality higher than the quality of $\boldsymbol{D}$, i.e., a task $a_{i}^{k}$ such that $q_{i}^{k}>p_{i}$.

Assume that at the time of making the purchase decision, the buyer's subjective assessment of the quality $p_{i}$ of $\boldsymbol{D}$ regarding her/his tasks is $\widehat{p}_{l}$. This assessment is based on the content of the message describing the product, which is provided by the seller. Buyer's purchase decision is based on the relationship between $q_{i}$ and $\widehat{p}_{l}$, where $q_{i}$ is the quality level the buyer is ready to pay for, say $c_{q}$. Final assessment of the correctness of the buyer's purchase decision depends on estimation of $\widehat{p}_{l}$ and the ordering of $p_{i}, q_{i}$ and $\widehat{p}_{l}$.

The utilization process, i.e., finding out the true properties of the product, brings $\widehat{p}_{l} \rightarrow p_{i}$ and the cost of this learning is denoted by $c_{u}$. This cost is smaller for "user friendly" products.

The quality needed to accomplish different tasks from category $A_{i}$ varies, i.e., for a certain quality level $p_{i}$ of $\boldsymbol{D}$, there are some tasks, which can be accomplished by using $\boldsymbol{D}$, and some tasks that the buyer will not be able to perform with $\boldsymbol{D}$. For the second group of tasks, the buyer has to find another way to accomplish them by paying extra for external service, denoted by

$C_{D}=\sum_{q_{i}^{k}>p_{i}} C_{i}^{k}$

The cost structure of the tasks' portfolio of $b$, is comprised by the price $c_{D}$ of the product $\boldsymbol{D}$ and the extra costs for external service $C_{D}$. Next, based on the above notations and discussion, we formulate an optimization problem to address buyer's optimal purchase decision.

\section{Optimization Problem}

The buyer's optimal purchase decision aims to minimize the following objective function over all available products on the market:

$$
\min _{D}\left(c_{D}+C_{D}+c_{u}+c_{\text {sunk }}\right),
$$

where $c_{\text {sunk }}$ is the cost of all preliminary actions, such as rejecting all inappropriate products, identifying tasks and related quality levels; and assessing the acceptable prices. We may ignore this cost, because it prepares for the decision making and it is not relevant in products' comparison. In function (1) the term $\mathrm{c}_{\text {sunk }}$ is a constant and will not affect the finding of the optimal product choice.

Each of the other three components of function (1) is composite and plays different role in the two phases of the new product adoption process:

- The price of the product $c_{D}$ can be considered also in relation to the cost of the tasks the

product $\boldsymbol{D}$ is suitable for, say $c=\sum_{q_{i}^{k}<p_{i}} c_{i}^{k}$ (this is the cost that could have need incurred for accomplishing these tasks without using $\boldsymbol{D})$, i.e., this is the buyer's benefit from having and using the product $\boldsymbol{D}$. The buyer's actual benefit is the difference $c_{b}=c-c_{D}$. The value of $c_{b}$ may change over the two phases of the product adoption process. During the acquiring phase the buyer is fully aware of the product's price and the tasks he/she intends to accomplish with $\boldsymbol{D}$, so denote the value of $c_{b}$ during the acquiring phase by $c_{b}^{1}$, i.e., $c_{b}^{1}$ is the initial measure of $c_{b}$. During the utilization phase this value may change because: (1) it may include costs of tasks the buyer initially (at the time of purchase) had not intended to accomplish with $\boldsymbol{D}$, but $\boldsymbol{D}$ is suitable for these tasks, and (2) it may exclude costs of tasks, which the buyer had intended to accomplish with $\boldsymbol{D}$, but $\boldsymbol{D}$ 
is not suitable for them. Denote the value of $c_{b}$ during the utilization phase by $c_{b}^{2}$, i.e., $c_{b}^{2}$ is the updated measure of $c_{b}$.

- The cost of the tasks, which the product is not suitable for is $C_{D}$. Here again, similarly to the previous discussion, we have initial and updated sets of tasks with corresponding $\operatorname{costs} C_{D}^{1}$ and $C_{D}^{2}$.

- In the case of making decision to place a warranty claim of misinforming leading to a replacement of the product (the initially purchased product $\boldsymbol{D}^{\mathbf{1}}$ proves to be unsuitable to the buyer) the utilization $\operatorname{cost} c_{u}$ will include the utilization of the replaced product $\boldsymbol{D}^{\mathbf{2}}$ or we may have again initial and updated utilization $\operatorname{costs} c_{u}^{1}$ and $c_{u}^{2}$.

Therefore, the optimal purchase decision will be based on maximizing the benefits, i.e., $\max _{D}\left(c_{b}\right)$ and minimizing the costs, i.e., $\min _{D}\left(C_{D}+c_{u}\right)$.

At the time of making the purchase decision, the buyer $b$ optimizes with respect of the initial measures $-\max _{\mathrm{D}}\left(c_{b}^{1}\right)$ and $\min _{\mathrm{D}}\left(C_{D}^{1}\right)$. The utilization cost $c_{u}$ is unknown at that time and it doesn't affect the decision making process.

Often during utilization phase, in the case of provided warranty of misinforming, the buyer faces a decision whether to make a warranty claim. This decision is based on solving again the maxmin problem, but now based on the updated measures and including the utilization cost. The utilization cost is important and may affect the buyer's decision of making a warranty claim leading to a product replacement. If this cost is too high, the buyer may infer that it will take a significant time and effort to utilize the newly obtained (via replacement) product and may decide not to make the claim and incur this cost again.

\section{Conclusion}

The role of the warranty of misinforming in new product adoption is very important as it provides protection for the customers to examine the product they are interested in through reassuring them that they can return the product if it is not suitable for their needs. At the same time it plays a promotional role for the seller because it attracts customers who are not entirely sure what they are looking for or their expertise does not allow for making a definite decision as to which product to buy. It allows customers to make purchase decisions close to the optimal according to a set of pre-specified criteria. Warranty of misinforming allows customers to assume the risk for purchasing a product with low acceptability. This increases the probability of making a correct, near optimal purchase decision under uncertainty.

The above observation on the utilization process reveals the following interesting fact: for products with high usability (especially learnability) the probability of making a claim under the warranty of misinforming is also high. One possible explanation of this fact is as follows: if the product has high usability, especially learnability, the customer gains expertise in using the product and advances her/his skills in utilizing not only this product, but more advanced products of the same range, which may lead to a decision to return the purchased product (which is acceptable under the warranty terms) and aim for a product with improved functionalities. Therefore, warranty of misinforming has an addition effect on the customers - it stimulates their learning and enhances their technical skills.

On the other hand, better understanding how a customer makes purchase decisions in acquiring a new product, the process and risks associated with the utilization of this product allows producers to provide more effective product service and support. The warranty of misinforming serves as a tool for collecting customers' feedback and plays a significant role in the overall improvement of customer service and customer satisfaction. 


\section{References}

Bhagat, S., Goyal, A., \& Lakshmanan, L.V.S. (2012). Maximizing product adoption in social networks. WSDM 2012 - Proceedings of the 5th ACM International Conference on Web Search and Data Mining, pp. 603-612.

Blischke, W., \& Murthy, D. N. P. (1996). Product warranty handbook. Marcel Dekker

Christozov, D., Chukova, S., \& Mateev, P. (2006). A measure of risk caused by information asymmetry in e-commerce. Issues in Informing Science and Information Technology, 3, 147-158.

Christozov, D., Chukova, S., \& Mateev, P. (2007). On the relationship between warranty and the risk of information asymmetry. Issues in Informing Science and Information Technology, 4, 235-249.

Christozov, D., Chukova, S., \& Mateev P. (2009). On two types of warranties: Warranty of malfunctioning and warranty of misinforming. Asia-Pacific Journal on Operation Research, 26(3), 399-420.

Christozov, D., Chukova, S., \& Mateev P. (2011). Assessment of risk of misinforming: Dynamic measures. Interdisciplinary Journal of Information, Knowledge, and Management, 6, 163-176.

Christozov, D., Chukova, S., \& Mateev, P. (2012). Market segmentation based on risk of misinforming reduction. Issues in Informing Science and Information Technology, 9, 253-265.

Devos, J., Van Landeghem, H., \& Deschoolmeester, D. (2012). SMEs and IT: Evidence for a market for "lemons". Electronic Journal Information Systems Evaluation, 15(1).

Eng, T. Y., \& Quaia, G. (2009). Strategies for improving new product adoption in uncertain environments: A selective review of the literature. Industrial Marketing Management, 38(3), 275-282.

Manchanda, P., Xie, Y., \& Youn, N. (2008). The role of targeted communication and contagion in product adoption. Marketing Science, 27(6), 961-976.

Parry, M. E., Cao, Q., \& Song, M. (2011). Forecasting new product adoption with probabilistic neural networks. Journal of Product Innovation Management, 28(SUPPL. 1), 78-88.

Sethi, S. P., Prasad, A., \& He, X. (2008). Optimal advertising and pricing in a new-product adoption model. Journal of Optimization Theory and Applications, 139(2), 351-360. 


\section{Biographies}

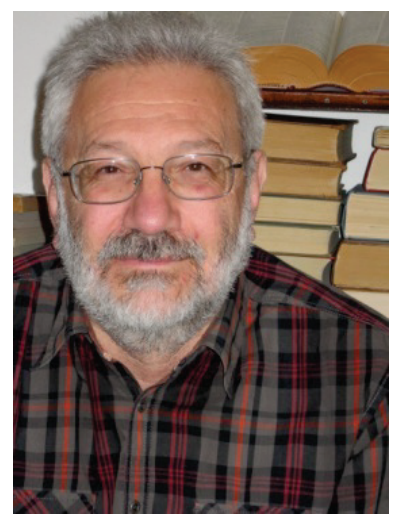

Dimitar Christozov is a Professor of Computer Science at the American University in Bulgaria. He has more than 30 years of experience in areas as computer science, quality management and information systems. He graduated Mathematics from Sofia University "St. Kliment Ohridski" in 1979. He completed his $\mathrm{PhD}$ thesis "Computer Aided Evaluation of Machine Reliability" in 1986. In 2009 he completes his D.Sc. thesis on "Quantitative Measures of Informing Quality". In ICTT "Informa" (1986-1993) Dr. Christozov was involved in establishing the national information network for technology transfer and conducted research in the areas of technologies assessment, integral quality measures and information systems for quality management. In these areas he was recognized as one of the leading experts in Bulgaria. Professor Christozov has more than 80 publications as separate volume, journal papers and papers in refereed proceedings. He is a founding member of Informing Science Institute and chair of Bulgarian Informing Science Society; and founding member of the Bulgarian Statistical Society.

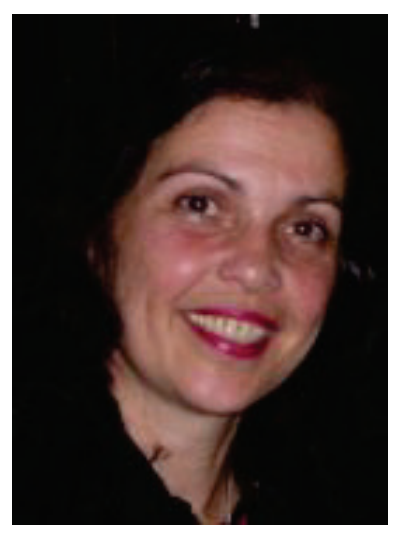

Dr. Stefanka Chukova is a Reader in Operations Research at the School of Mathematics, Statistics and Operation Research, Victoria University of Wellington, Private Bag 600, Wellington, New Zealand, e-mail: schukova@msor.vuw.ac.nz. She has a PhD in Mathematics (concentration in Probability and Statistics) from University of Sofia, Sofia, Bulgaria. Her research interests are in applied stochastic models, warranty analysis, reliability and queuing. She has more than 80 publications and has presented papers at national and international conferences. Stefanka is an elected member of ISI.

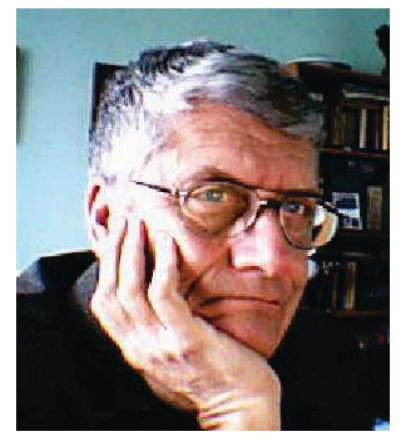

Dr. Plamen Mateev is Associate Professor and Supervisor of Statistical laboratory at the Department "Probability, Operation Research, Statistics", in Sofia University "St.Kliment Ohridski", Faculty of Mathematics and Informatics, Bulgaria, 1164 Sofia, 5, J. Boucher Str., e-mail: pmat@,fmi.uni-sofia.bg.

He is a fellow of the Institute of Mathematics and Informatics of the Bulgarian Academy of Sciences.

His MSc in Mathematical Statistics is from Sofia University and his $\mathrm{PhD}$ is from Moscow State University. The research interests are in communication theory, applied statistics, statistical software and applications. He has published nearly 100 papers in scientific journals and conference proceedings. He was the Chair of Bulgarian Statistical Society and a member of ENBIS, IBS and a founding member of the Bulgarian Informing Science Society. 